\title{
ELABORAÇÃO DE PROJETO DE SPDA, EM GALPÃO FABRIL NO ESTADO DE MINAS GERAIS
}

André Luís da Silva Pinheiro

Doutor em Engenharia Nuclear pela Universidade Federal do Rio de Janeiro (UFRJ)

Rio de Janeiro, RJ, Brasil

apinheiro99@gmail.com

Antônio José Dias da Silva

Mestre em Sistemas de Informação pela Universidade Federal do Rio de Janeiro (UFRJ) Professor do Centro Universitário Augusto Motta - UNISUAM

Rio de Janeiro, RJ, Brasil antoniojoseds@gmail.com

Geraldo Motta Azevedo Júnior Doutor em Engenharia Elétrica pela Universidade Federal do Rio de Janeiro (COPPE/UFRJ) Professor do Centro Universitário Augusto Motta - UNISUAM

Rio de Janeiro, RJ, Brasil geraldomotta@unisuam.edu.br

Adenoci Carvalho dos Santos Neto

Graduando em Engenharia Elétrica pelo Centro universitário Augusto Motta (UNISUAM) Rio de Janeiro, RJ, Brasil adenoci.c@gmail.com

\section{RESUMO}

O presente trabalho se destina a abordar de forma técnica, a implementação de um sistema de proteção contra descargas atmosféricas (SPDA) de um galpão fabril situado na cidade de São João Nepomuceno em Minas Gerais. Tal edificação abriga uma indústria têxtil que, em visita feita a área da edificação, observou-se que o galpão não possui nenhuma forma de proteção contra descargas atmosféricas. Sendo assim, neste trabalho, será feita uma breve revisão da origem do fenômeno da descarga atmosférica até a captação, descida, aterramento e equipotencialização do imóvel atingido pelo raio. Também será motivo de estudo, os cálculos necessários para avaliação das instalações do SPDA assim como os métodos usuais de SPDA. Através da análise de riscos, pode-se comprovar que o imóvel apresenta valores elevados de risco, principalmente nas componentes RA e RU (ferimentos em seres vivos), justificando assim sua implementação. A presente obra ainda sugere como custo de projeto o valor de $\mathrm{R} \$ 21.167,66$ que se mostra inferior ao valor que uma possível falha poderia custar, tendo em vista o valor do maquinário, dos materiais utilizados e da paralização da produção. Desta forma, a proposta do projeto do SPDA para o galpão fabril, se mostra adequada e viável, reduzindo o risco existente das descargas elétricas à valores aceitáveis, assegurando assim a proteção aos seres vivos e ao imóvel.

Palavras-chave: SPDA, Análise de risco, Descargas atmosféricas, Para-raios, Proteção

\section{PREPARATION OF A SPDA PROJECT, IN A MANUFACTURING WAREHOUSE IN THE STATE OF MINAS GERAIS}

\section{ABSTRACT}

The present work aims to approach in a technical way, the implementation of a lightning protection system (SPDA) of a factory building located in the city of São João Nepomuceno in Minas Gerais. 
This building houses a textile industry that, on a visit made to the building area, it was observed that the shed does not have any form of protection against lightning strikes. Therefore, in this work, a brief review of the origin of the phenomenon of atmospheric discharge until the capture, descent, grounding, and equipotentialization of the property affected by the lightning will be made. It will also be a matter of study, the calculations necessary to evaluate the facilities of the SPDA as well as the usual methods of SPDA. Through the risk analysis, it can be verified that the property presents high-risk values, mainly in the RA and RU components (injuries to living beings), justifying its implementation. The present work still suggests a project cost of $R \$ 21,167.66$, which is lower than the amount that a possible failure could cost, considering the value of the machinery, materials used, and production stoppage. In this way, the SPDA's project proposal for the factory building is adequate and feasible, reducing the risk of electric discharges to acceptable values, thus ensuring protection for living beings and property.

Keywords: SPDA, Risk analysis, Atmospheric discharges, Lightning rod, Protection

\section{INTRODUÇÃO}

\subsection{Metodologia}

Este trabalho irá adotar a seguinte metodologia: Em seu primeiro momento, foi feita de uma pequena análise de como o fenômeno das descargas atmosféricas se formam, em seguida, uma breve análise das principais formas de proteção contra descargas atmosféricas, seus tipos e materiais mais usados na elaboração de um SPDA. Ao fim dessa primeira parte será analisado as principais técnicas de Sistema de Proteção Contra Descargas Atmosféricas e se tais especificações podem ser aplicadas ao projeto. Em seguida, será feita uma análise e gerenciamento dos riscos envolvidos no projeto. Ao fim será apresentado o projeto do SPDA vem como uma planta baixa da edificação, com exemplos e detalhes técnicos.

\subsection{Revisão Bibliográfica}

Com o passar dos anos o aquecimento global (VEIGA, 2008) vem se confirmando como uma realidade, alterando o clima de nosso planeta muito rapidamente, com isso vemos que as estações do ano estão cada vez mais severas, o que acaba agravando um fenômeno natural que nos dias de hoje ocorre com cada vez mais frequência, as descargas atmosféricas (FILHO, 2005).

Após uma visita a um galpão fabril situado no estado de Minas Gerais, na cidade de São João Nepomuceno, pode-se constatar que tal galpão não possui nenhum sistema de proteção contra descargas atmosféricas, comprometendo assim a segurança tanto das máquinas quanto dos funcionários do local. O presente trabalho, destina-se a abordar de forma técnica, a implementação de um sistema de proteção contra descargas atmosféricas (SPDA) (CREDER, 2016) e assim, minimizar 
os danos, tanto das perdas de capital financeiro, quanto a perda inestimável de vidas humanas decorrentes das descargas atmosféricas.

O Brasil é o país com maior incidência de descargas atmosféricas (FILHO, 2005), é líder mundial na incidência deste fenômeno. Segundo o Grupo de Eletricidade Atmosférica (ELAT http://ww.inpe.br/elat/) do Instituto Nacional de Pesquisas Espaciais (INPE), este fenômeno provocou entre 2000 e 2019 a morte de 2.194 pessoas ou seja, a cada 50 mortes por raio no mundo uma acontece no Brasil, conforme ilustrado no infográfico de mortes por raio no Brasil do ELAT (Figura 1). Ainda neste mesmo infográfico, Minas Gerais teve a maior incidência de raios em uma tempestade, foram mais de 180 descargas por hora, isso significa que o Estado mineiro concentrou $16 \%$ dos raios dissipados no território nacional e $74 \%$ das descargas atmosféricas que incidiram entre os quatro estados da região sudeste, de acordo com dados da USP. Segundo o INPE e a PUCMG é normal um aumento dos raios durante a primavera e o verão, aumentando também o número de mortes em decorrência desses eventos.

Figura 1 - Infográfico de mortes por raio no Brasil

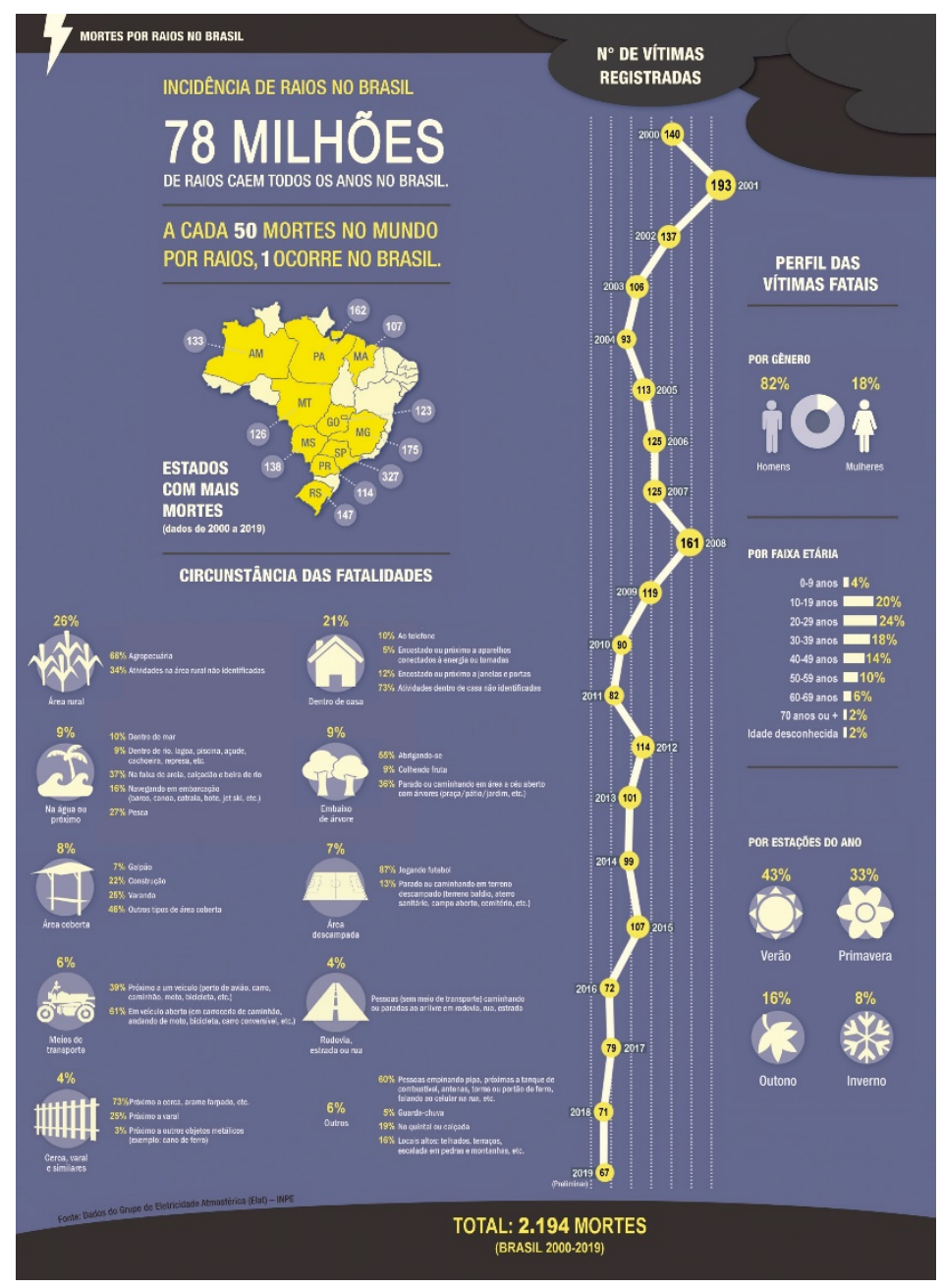

Fonte: Adaptado de http://www.inpe.br/webelat/imagesNovoLayout/arte/Infografico_Mortes_Raios_20002019_alta.jpg, acessado em 03/06/2019 
Apesar de parecer singelo, para a ciência moderna, o fenômeno conhecido como descarga atmosférica ou simplesmente raio, passou a maior parte da história como uma incógnita para a humanidade, sendo seu conceito elétrico admitida pelo mundo acadêmico só em meados do século XVIII, graças ao sucesso dos experimentos de Thomas-François Dalibard, na França, e de Benjamin Franklin, nos Estados Unidos, podendo assim abordar novos conceitos sobre esse fenômeno. Os raios surgem por um processo de eletrização das nuvens de chuva, tais nuvens são definidas como nuvens de tempestade, e também podem ser chamada de nuvens Cumulunimbus (Cb) (Figura 2), porém outros tipos de nuvens podem ocasionar relâmpagos e trovões (MATTOS, 2009).

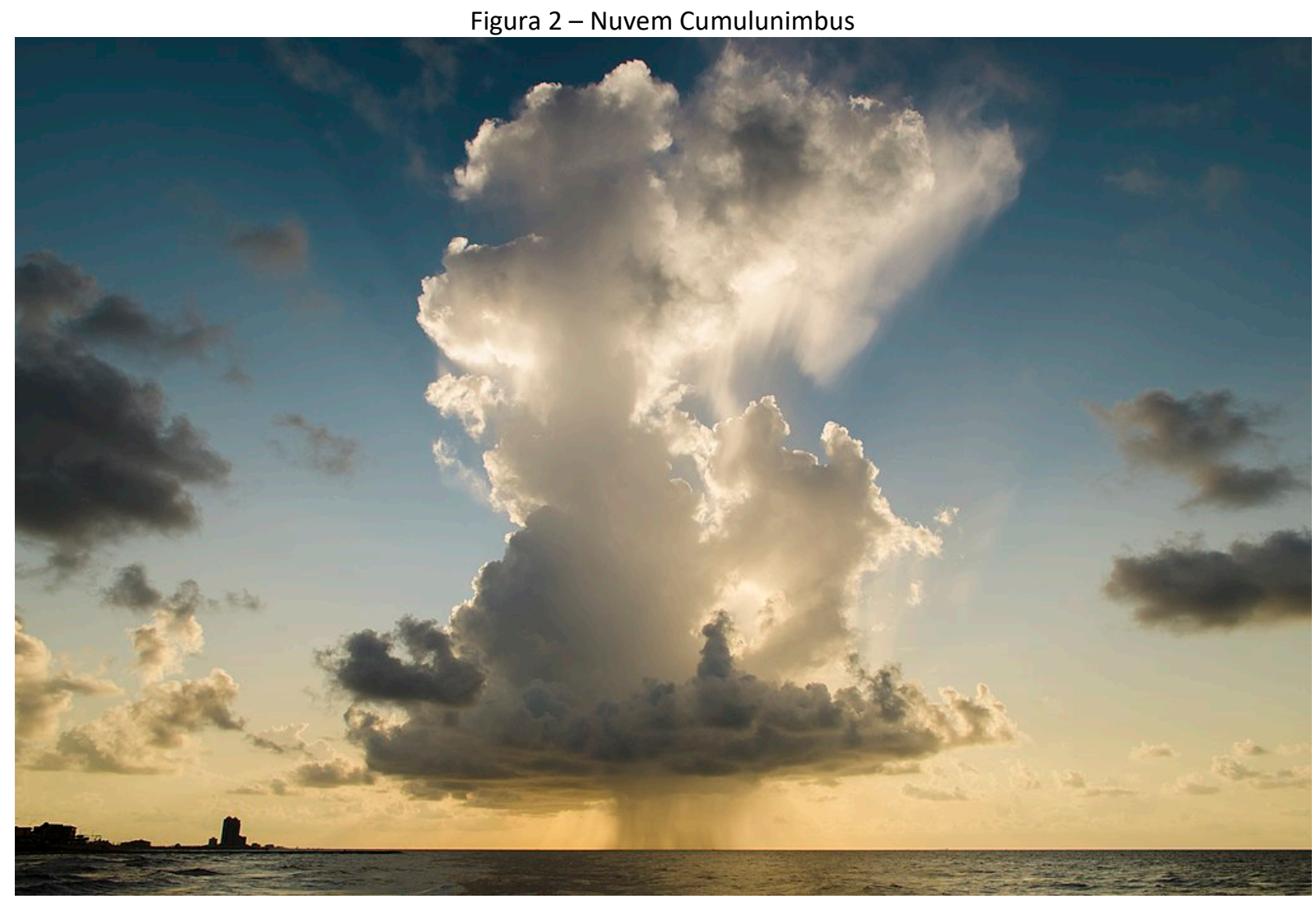

Adaptado de https://www.wikiwand.com/en/Cumulonimbus_calvus, acessado em 04/06/2019

Tempestades de areia, neve e erupções vulcânicas, podem também produzir mesmo que em menor escala, tal fenômeno. A eletrização das nuvens de chuva (FILHO, 2005), se dá através da formação e movimentação das cargas elétricas no seu interior.

Assim como as cargas elétricas de mesmo sinal se repelem, as cargas elétricas de sinais opostos são atraídas. Portanto, as cargas negativas na nuvem tenderiam a se aproximar das cargas positivas na superfície da Terra, mas devido ao ar ser um isolante elétrico, ele não permite que as cargas se aproximem. No entanto, à medida que as concentrações de cargas elétricas iguais vão aumentando, o campo elétrico próximo a elas se torna mais intenso. 
Os campos elétricos de alta intensidade podem liberar alguns elétrons dos átomos do ar, e são esses elétrons livres que ajudam a conduzir a corrente elétrica. Quando esse fenômeno ocorre, as cargas elétricas opostas da superfície da Terra e das partes mais baixas das nuvens podem finalmente se encontrar, sendo esse encontro chamado descarga elétrica atmosférica, ou mais comumente, raios. A Figura 3 exemplifica graficamente como os raios se formam devido as cargas elétricas.

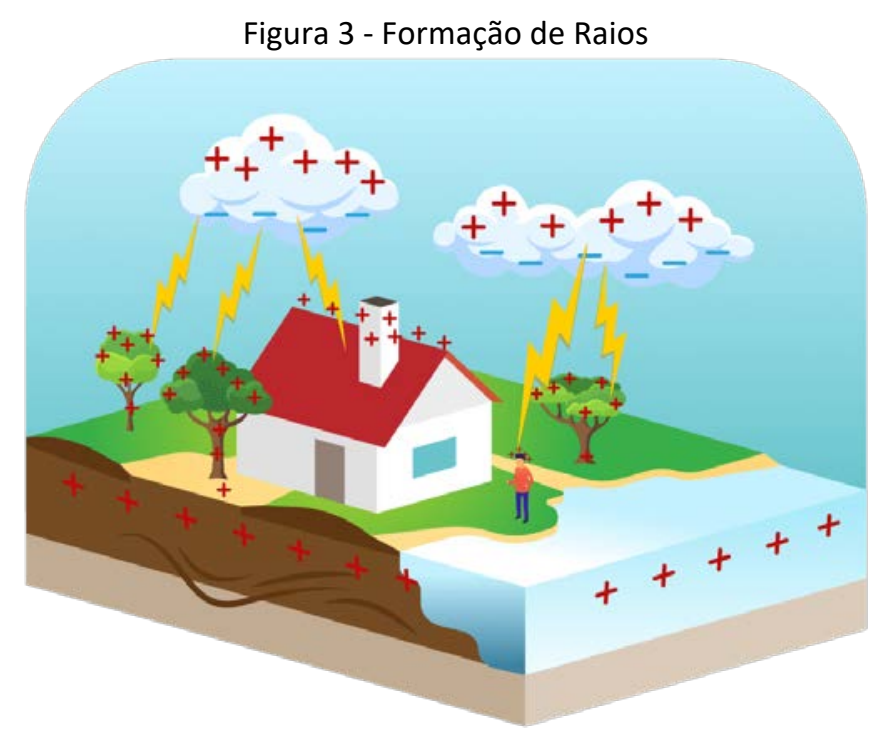

Fonte: Adaptado de https://blog.biologiatotal.com.br/raios-trovoes-e-relampagos/, acessado em 06/06/2019

Segundo a conclusão do Grupo de Eletricidade Atmosférica (ELAT) e do Instituto Nacional de Pesquisas Espaciais (INPE) (ARAÚJO, 2010), a energia elétrica nos últimos anos está cada vez mais atrelada ao desenvolvimento econômico das nações e o desenvolvimento industrial caminha lado a lado com os avanços tecnológicos. Desta forma, os sistemas de proteção também evoluem para proteger homem e máquina. Estima-se que o Brasil tem um prejuízo na casa dos milhões de reais todos os anos, decorrentes das descargas atmosféricas que atingem os campos fabris isso se levarmos em conta só o setor elétrico. Se expandirmos a visão, essa cifra vai para a casa dos bilhões de dólares pois, estamos falando de perdas em todos os seguimentos da indústria inclusive, paralização da produção. Posto isto, o Sistema de Proteção Contra Descargas Atmosféricas (SPDA) (CREDER, 2016) vem para minimizar tais efeitos adversos causado pelos raios, protegendo bens imóveis e as áreas no seu entorno.

O critério que indica o número de dias de trovoada por ano em uma determinada região, é chamado índice cerâunico. Este parâmetro é mais realista quando decorre por um longo período pois, essa observação registra os trovões dentro de uma localização geralmente em um círculo com raio de 20 quilômetros. Registrando em um único mapa todos os acontecimentos consegue-se ligar 
os pontos de igual intensidade e assim obtém-se as linhas de mesmo índice, ou seja, os índices isocerâunicos. Pode-se ver o mapa isocerâunico do Brasil na Figura 4

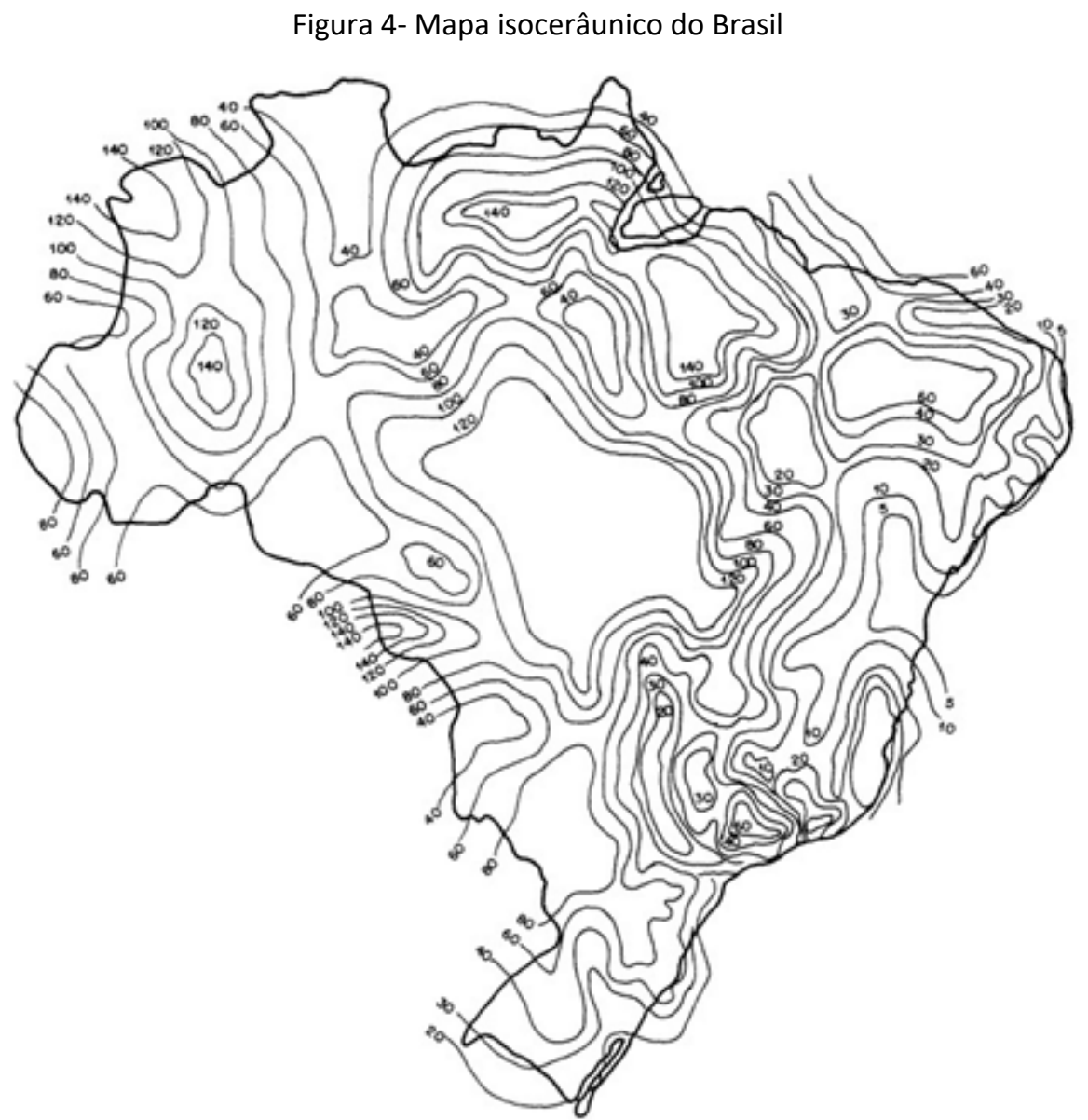

Fonte: Adaptado de http://construdicasjbasc.blogspot.com/2015/05/eletrica-sistema-spda-parte-1.html, acessado em $07 / 06 / 2019$

O SPDA é um sistema integral de proteção destinado a proteger uma construção ou estrutura contra descargas atmosféricas, tradicionalmente tem sido chamado de para-raios. O sistema tem por objetivo blindar a edificação, seus ocupantes e todos os conteúdos no interior da estrutura dos efeitos térmicos, mecânicos e elétricos associados aos relâmpagos. O SPDA atua de maneira que a descarga elétrica possa entrar e sair sem passar pelas partes condutoras da edificação ou dos seus ocupantes, evitando assim danos ou acidentes. Um para-raios não impede que relâmpagos atinjam a estrutura, ele constrói um caminho para que a corrente elétrica presente no raio flua do ponto de captação até o solo. Tal ideia foi formulada a séculos atrás por Benjamin Franklin (Figura 5), com seu experimento da pipa na tempestade (MINISTÉRIO DA CIDADANIA, 2019), (ELAT, 2019). 
Figura 5 - Benjamin Franklin e seu experimento da pipa na tempestade

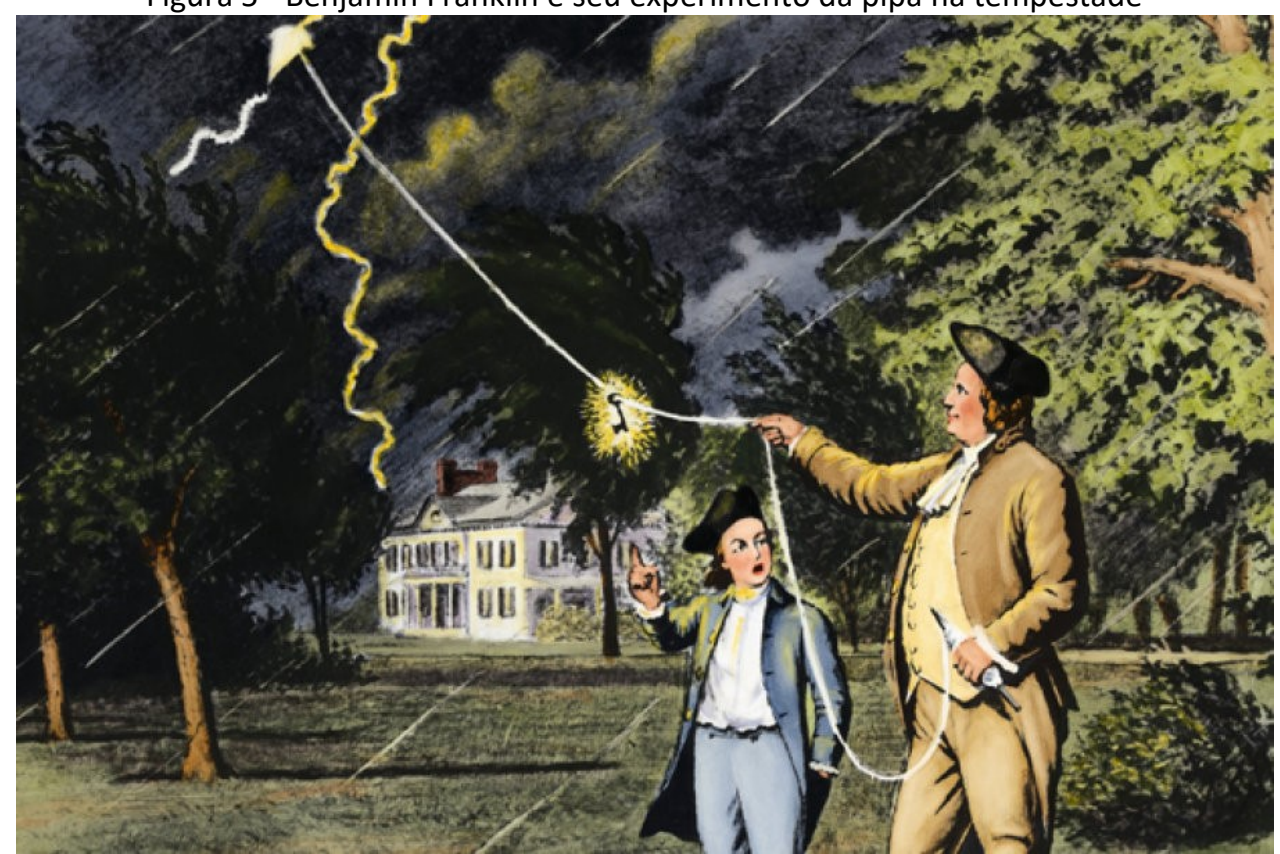

Adaptado de https://museuweg.net/blog/benjamin-franklin-ciencia-e-eletricidade/, acessado em 07/06/2019

O método de Franklin se fundamenta no princípio que uma descarga piloto descendente poder ser capturada por uma descarga ascendente, que decorre pelos captores instalados na edificação, esses captores podem ser hastes metálicas, cabos ou até mesmo elementos naturais. De tal forma que a corrente elétrica proveniente do relâmpago seria dissipada no solo através do SPDA, e não através do objeto a que se deseja proteger.

Nesse tipo de sistema a concentração de carga presente no solo da edificação vai migrando para o sistema de captação, que se encontra no topo da edificação, desta forma o líder descendente (relâmpago), atrai o líder ascendente (carga presente no captor), gerando um sistema de atração mútua, conforme exemplificado graficamente na Figura 6 (STÉFANI, 2011).

Figura 6- Método de Franklin
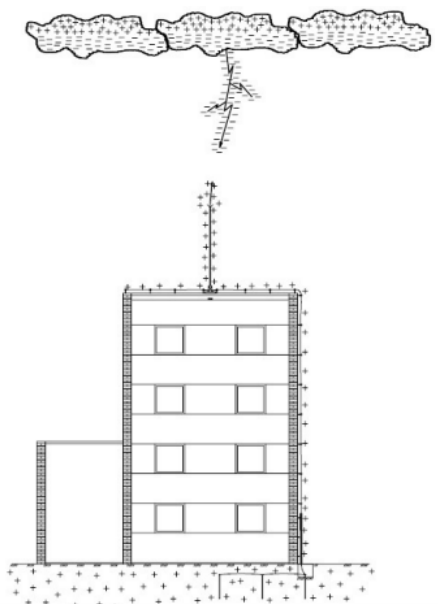

Fonte: (STÉFANI, 2011) 
O método de Franklin é muito recomendado para estruturas mais elevadas e de pouca área horizontal, pois desta forma pode-se utilizar uma menor quantidade de captores, o que torna o projeto mais viável economicamente. Esse método recebeu o nome de seu inventor, o físico e químico inglês Michael Faraday (1791 - 1876) (FRAZÃO, 2019), realizando experimentos ele descobriu que ao envolver um volume por uma gaiola metálica, este ficava blindado dos campos magnéticos, observou-se que ao aterrar a gaiola de Faraday, como se pode ver na Figura 7, as descargas elétricas induzidas por um líder ascendente sobre um líder descendente se dava por intermédio das partes metálicas da gaiola de Faraday. Desta forma a corrente elétrica circula pela gaiola, e blinda o que está dentro. (STÉFANI, 2011)

Figura 7 - Método da gaiola de Faraday

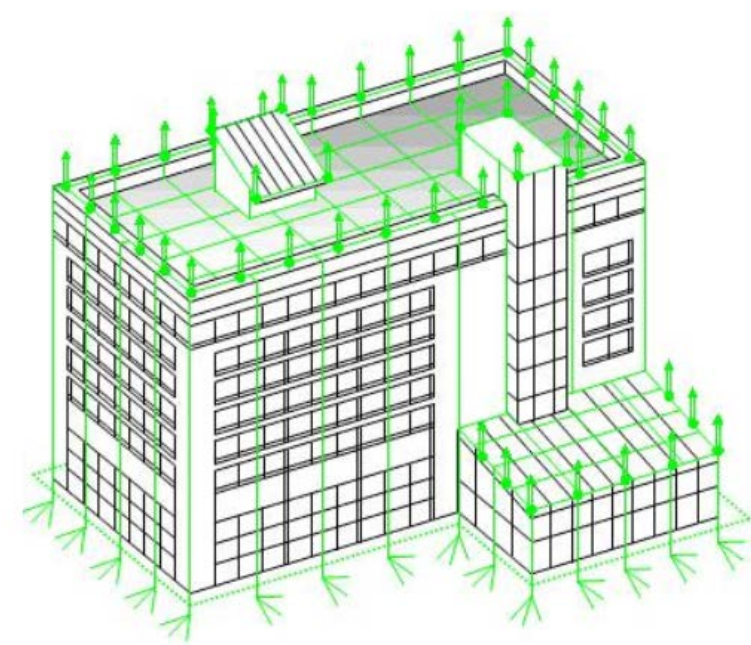

Fonte: Adaptado de (VAZQUEZ, 2018)

Já o método esfera-rolante, que tem o conceito de raio de atração, é um pouco mais complexo por ser mais abstrato. Trata-se da distância estimada em que a descarga descendente e ascendente se conectam com uma certa probabilidade, onde os captores são posicionados de maneira que qualquer líder descendente, que apareça nas imediações do volume de proteção estaria a metros do captor. Esse sistema foi criado prioritariamente para a proteção de redes de transmissão e se baseia em modelagem matemática, estudos fotográficos, ensaios em laboratório de alta tensão, medição de parâmetros dos raios, entre outros. (NOLETO, 2006) 


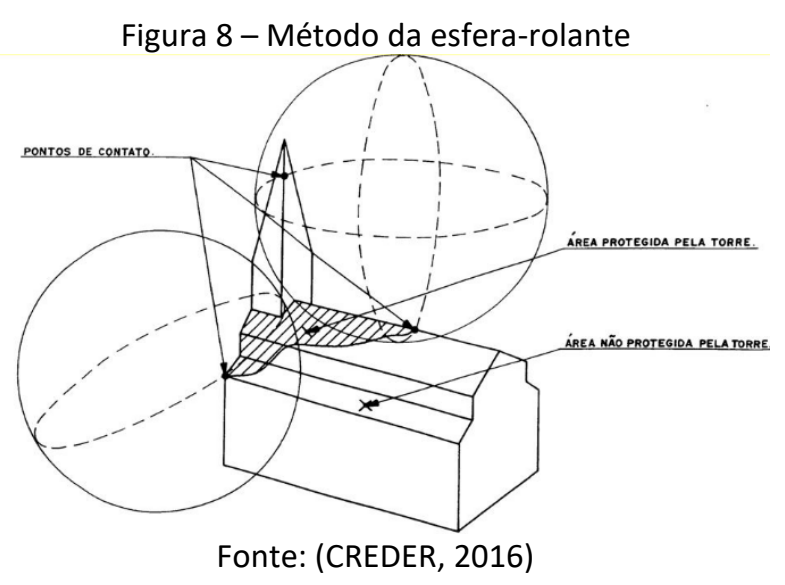

Em um sistema elétrico, é de suma importância que os aspectos técnicos sejam respeitados para que assim sejam mínimos os índices de falhas. Com isso é necessário a execução de um bom sistema de aterramento, que busca sempre a menor resistência e de maneira homogênea. Esse sistema tem por objetivo, obter uma resistência de aterramento mais baixa possível para as correntes de falta à terra, mantendo assim os potenciais decorrentes de correntes de falta, dentro dos limites de segurança. Podemos então dizer que algumas das funções dos sistemas de aterramento são: Proteger a vida, evitando a fibrilação do coração, escoar as cargas estáticas geradas nas carcaças dos equipamentos e elaborar um caminho para escoar para a terra as correntes produzidas pelo raio (KINDERMANN, 1995).

A elaboração e implementação de um projeto de aterramento que satisfaça a norma é, muitas vezes, um enorme desafio visto as condições da natureza do local, pois há muita variação de resistência de acordo com o tipo do solo, regiões rochosas possuem uma alta resistividade, por causa das características de sua composição mineral, que acaba por dificultar a obtenção de uma baixa resistividade, necessária a malha de aterramento para que assim satisfaça o projeto.

\section{DESENVOLVIMENTO}

\subsection{Análise e Gerenciamento de Riscos}

O risco é um valor a partir do qual se estabelece uma provável perda anual média de vidas ou bens na estrutura a ser protegida devido as descargas atmosféricas. A análise de riscos visa determinar quando é necessário, e em qual medida deve ser adotado um sistema de proteção, a fim de minimizar as perdas em decorrência dos raios. Esses riscos estão diretamente ligados: ao número anual de descargas atmosféricas que influenciam a estrutura; a probabilidade de dano por uma das descargas atmosféricas e a quantidade média das perdas causadas (SUETA, 2015). 
A (NBR 5419, 2015) codifica a análise em três tópicos, são eles: fontes de danos, tipos de danos e tipo de perdas, assim facilitando seu entendimento.

A principal fonte de danos tem sua origem na corrente da descarga atmosférica, e a sua severidade está associada ao ponto de impacto, esses são divididos em:

- S1: descarga atmosférica que atinge a estrutura;

- S2: descarga atmosférica que atinge áreas próximas à estrutura;

- S3: descarga atmosférica que atinge a linha de energia elétrica, linha telefônica e cabo de internet;

- S4: descarga atmosférica que atinge as proximidades da linha de energia elétrica, linha telefônica e cabo de internet.

Os danos causados por uma descarga atmosférica estão associados ao tipo de construção, os riscos a serem considerados são:

- D1: ferimentos a seres vivos por choque elétrico;

- D2: danos físicos;

- D3: falhas de sistemas eletroeletrônicos.

Os tipos de perdas a serem considerados são:

- L1: ferimentos a seres vivos por choque elétrico;

- L2: perda de serviço público;

- L3: perda de patrimônio cultural;

- L4: perdas de valores econômicos (estrutura, os bens nela contidos e perda de atividade desenvolvida na edificação).

O risco para ser avaliado deve levar em consideração, um valor relativo a uma provável perda anual média. O risco resultante deve ser avaliado, e para isso temos as seguintes questões a considerar:

- R1: risco de perda de vida humana (incluindo ferimentos permanentes);

- R2: risco de perda de serviço ao público;

- R3: risco de perda de patrimônio cultural;

- R4: risco de perda de valores econômicos.

Cada risco é a soma de seus componentes de risco. Ao calcular um risco, os componentes de risco podem ser agrupados de acordo com as fontes de danos e os tipos (NBR 5419, 2015).

Os componentes de risco (Tabela 1) para uma estrutura são divididos em: RA, RB, RC, RM, $R U, R V, R W$ e RZ, e esses são somados para que se tenha um valor de risco definido. 
Tabela 1 - Fatores que influenciam os componentes de risco

\begin{tabular}{|c|c|c|c|c|c|c|c|c|}
\hline $\begin{array}{l}\text { Características da estrutura ou dos sistemas } \\
\text { Internos (medidas de proteção) }\end{array}$ & RA & RB & $\mathrm{RC}$ & RM & $R U$ & RV & RW & $\mathrm{RZ}$ \\
\hline Área de exposição equivalente & $\mathrm{X}$ & $\mathrm{X}$ & $\mathrm{X}$ & $X$ & $\mathrm{X}$ & $\mathrm{X}$ & $\mathrm{X}$ & $\mathrm{X}$ \\
\hline Resistividade da superfície do solo & $\mathrm{X}$ & & & & & & & \\
\hline Resistividade do piso & $\mathrm{X}$ & & & & $\mathrm{X}$ & & & \\
\hline $\begin{array}{l}\text { Restrições físicas, isolamento, avisos visíveis, } \\
\text { equipotencialização do solo }\end{array}$ & $\mathrm{X}$ & & & & $x$ & & & \\
\hline SPDA & $\mathrm{X}$ & $\mathrm{X}$ & $\mathrm{X}$ & $\mathrm{Xa}$ & $\mathrm{Xb}$ & $\mathrm{Xb}$ & & \\
\hline Ligação ao DPS & $\mathrm{X}$ & $\mathrm{X}$ & & & $\mathrm{X}$ & $\mathrm{X}$ & & \\
\hline Interfaces isolantes & & & Xc & $\mathrm{Xc}$ & $x$ & $\mathrm{x}$ & $\mathrm{X}$ & $\mathrm{x}$ \\
\hline Sistema coordenado de DPS & & & $\mathrm{x}$ & $\mathrm{x}$ & & & $\mathrm{X}$ & $\mathrm{X}$ \\
\hline Blindagem espacial & & & $\mathrm{X}$ & $\mathrm{X}$ & & & & \\
\hline Blindagem de linhas externas & & & & & $\mathrm{X}$ & $\mathrm{X}$ & $\mathrm{X}$ & $\mathrm{X}$ \\
\hline Blindagem de linhas internas & & & $\mathrm{X}$ & $\mathrm{X}$ & & & & \\
\hline Precauções de roteamento & & & $\mathrm{X}$ & $\mathrm{X}$ & & & & \\
\hline Sistema de equipotencialização & & & $\mathrm{X}$ & & & & & \\
\hline Precauções contra incêndios & & $\mathrm{X}$ & & & & $\mathrm{X}$ & & \\
\hline Sensores de fogo & & $\mathrm{X}$ & & & & $\mathrm{X}$ & & \\
\hline Perigos especiais & & $\mathrm{X}$ & & & & $\mathrm{X}$ & & \\
\hline Tensão suportável de impulso & & & $\mathrm{X}$ & $\mathrm{X}$ & $\mathrm{X}$ & $\mathrm{X}$ & $x$ & $\mathrm{X}$ \\
\hline \multicolumn{9}{|l|}{$\begin{array}{l}\text { a } \rightarrow \text { Somente para SPDA tipo malha externa. } \\
b \rightarrow \text { Devido a ligações equipotenciais. } \\
c \rightarrow \text { Somente se eles pertencem ao equipamento. }\end{array}$} \\
\hline
\end{tabular}

Fonte: (NBR 5419, 2015)

Para esse trabalho foi utilizado os tipos de riscos R1 e R4, porém o risco de perda de valores econômicos não será abordado no transcorrer da análise por ser facultativo de acordo com a norma vigente. Os componentes de risco utilizados serão o $R A, R B, R U$ e RV, os outros quatro não serão utilizados por tratar exclusivamente de áreas hospitalares e ambientes explosivos.

\subsubsection{Determinação das quantidades relevantes e zonas de proteção}

O primeiro passo para que se possa avaliar o risco presente em uma edificação é identificar quais perdas estão associadas a ela e em qual zona, para esse trabalho identificou-se que a zona de risco compreende apenas a área interna da edificação, uma vez que os funcionários entrarão para trabalhar no interior da galpão e sairão ao término do expediente de trabalho. As perdas que serão avaliadas são:

- L1: ferimentos a seres vivos por choque elétrico;

- L4: perdas de valores econômicos.

Com os tipos de perdas definidas, é necessário avaliar a necessidade de implementação de um sistema de proteção. Os riscos associados a essas perdas são:

- R1: risco de perda de vida humana, incluindo ferimentos;

- R4: risco de perda de valores econômicos. 
Os componentes presentes em R1, são: RA, RB, RU e RV.

Os componentes presentes em R4, são: RB, RC, RM, RV, RW e RZ.

Depois de calculados os riscos seus valores devem ser comparados com os descritos na Tabela 2, para saber a real necessidade de um sistema de proteção.

Tabela 2 - Valores típicos de risco tolerável $R_{T}$

\begin{tabular}{c|l|c}
\hline \multicolumn{2}{c|}{ Tipo de perda } & $\boldsymbol{R}_{\boldsymbol{T}}\left(\boldsymbol{y}^{-\mathbf{1}}\right)$ \\
\hline L1 & Perda de vida humana ou ferimentos permanentes & $10^{-5}$ \\
\hline L2 & Perda de serviço ao público & $10^{-3}$ \\
\hline L3 & Perda de patrimônio cultural & $10^{-4}$ \\
\hline
\end{tabular}

Fonte: adaptado de (NBR 5419, 2015)

O risco de perda de valor econômico não será calculado, pois de acordo com a norma esse é facultativo. Para que essa análise fosse feita foi criada tabelas com as informações relevantes do projeto e para análise de riscos.

A Tabela 3 foi criada para reunir todos os dados pertinentes a análise de riscos, assim facilitando o seu entendimento e servindo de base para todos os cálculos que virão a seguir.

Tabela 3 - Dados gerais da edificação

\begin{tabular}{|c|c|c|c|}
\hline Parâmetro de entrada & Comentário & Símbolo & Valor \\
\hline Comprimento da estrutura & em metros & $\mathrm{L}$ & 58,34 \\
\hline Largura da estrutura & em metros & W & 24,83 \\
\hline Altura da estrutura & em metros & $\mathrm{H}$ & 6,00 \\
\hline Índice cerâunico & médio & $N_{G}$ & 5,20 \\
\hline Fator de localização & estrutura isolada & $C_{D}$ & 1,00 \\
\hline Fator inst. Linha & aérea & $C_{I}$ & 1,00 \\
\hline Fator tipo de linha & N/A & $C_{T}$ & 1,00 \\
\hline Fator ambiental & suburbano & $C_{E}$ & 0,50 \\
\hline Comprimento da linha elétrica & em metros & $L_{L}$ & 1000 \\
\hline Comprimento da linha telecom. & em metros & $L_{L}$ & 1000 \\
\hline SPDA & não possui & $P_{B}$ & 1,00 \\
\hline Proteção contrachoque & estrutura: N/A & $P_{T A}$ & 1,00 \\
\hline Proteção contrachoque & linha: N/A & $P_{T U}$ & 1,00 \\
\hline Ligação equipotencial & sem DPS & $P_{L D}$ & 1,00 \\
\hline Fator de blindagem & não blindada & $C_{L D}$ & 1,00 \\
\hline Fator de blindagem & não blindada & $C_{L I}$ & 1,00 \\
\hline L1: tensão de toque e passo & todos os tipos & $L_{T}$ & $10^{-2}$ \\
\hline L1: devido à danos físicos & indústria & $L_{F}$ & $2 \times 10^{-2}$ \\
\hline Superfície do piso & concreto & $r_{t}$ & $10^{-2}$ \\
\hline Tempo na estrutura & por pessoa & $t_{z}$ & 6000 \\
\hline Número de pessoas & zona de proteção & $n_{z}$ & 100 \\
\hline Número de pessoas & em toda estrutura & $n_{t}$ & 100 \\
\hline
\end{tabular}




\begin{tabular}{l|l|c|c}
\hline Proteção de incêndio & não possui & $r_{p}$ & 1,00 \\
\hline Risco de incêndio & baixo & $r_{f}$ & $10^{-3}$ \\
\hline L1: perigo especial & não possui & $h_{z}$ & 1,00 \\
\hline
\end{tabular}

Fonte: (Os autores, 2019)

\subsection{2. Área de exposição equivalente e número anual de eventos perigosos}

A área de exposição equivalente, $A_{D}$, pode ser calculada de acordo com a expressão (1), essa utilizada para estruturas retangulares, isoladas e simples. Ao se substituir os valores na equação, o valor obtido foi $5460,58 m^{2}$

$$
A_{D}=L \cdot W+2 \cdot(3 \cdot H) \cdot(L+W)+\pi \cdot(3 \cdot H)^{2}
$$

Onde:

$A_{D}$ é a área de exposição equivalente em $\mathrm{m}^{2}$;

L é o comprimento da estrutura em metros;

W é a largura da estrutura em metros;

H é a altura da estrutura em metros.

O número de eventos perigosos para estrutura, $N_{D}$, é expresso pela equação (2). E ao substituirmos os dados temos $N_{D}$ igual a 0,0284 .

$$
N_{D}=N_{G} \cdot A_{D} \cdot C_{D} \cdot 10^{-6}
$$

Onde:

$N_{D}$ é o número de eventos perigosos;

$N_{G}$ é a densidade de descargas atmosféricas para a terra $\left(1 / \mathrm{km}^{2} \mathrm{x}\right.$ ano);

$A_{D}$ é a área de exposição equivalente em $\mathrm{m}^{2}$;

$C_{D}$ é o fator de localização da estrutura.

O número de eventos perigosos para estruturas adjacentes, $N_{D J}$, não se aplica nesse caso, pois a edificação está isolada das demais. A avaliação do número médio anual de eventos perigosos devido a descargas atmosféricas perto da estrutura, $N_{M}$, é expressa pela equação (3), e o termo $A_{M}$, que se refere a área de exposição equivalente de descargas atmosféricas, e é encontrado com a equação (4).

$$
N_{M}=N_{G} \cdot A_{M} \cdot 10^{-6}
$$

Onde:

$N_{M}$ é a avaliação do número médio anual de eventos perigosos; 
$N_{G}$ é a densidade de descargas atmosféricas para a terra $\left(1 / \mathrm{km}^{2} \mathrm{x}\right.$ ano);

$A_{M}$ é a área de exposição equivalente de descargas atmosféricas que atingem perto da estrutura, em $\mathrm{m}^{2}$, é expressa pela equação (4).

$$
A_{M}=2.500 .(L+W)+\pi .500^{2}
$$

Onde:

L é o comprimento da estrutura em metros;

W é a largura da estrutura em metros.

Ao se calcular os parâmetros substituindo os valares nas equações (3) e (4) foram encontrados os seguintes resultados 4,5166 e $868568,16 \mathrm{~m}^{2}$ respectivamente. A avaliação do número médio anual de eventos perigosos na linha devido as descargas atmosféricas, $N_{L}$, é calculada utilizando a expressão (5). Desta forma, encontra-se o valor de $N_{L}$ igual a 0,104, admitindo que o comprimento da linha de telecomunicações será igual ao comprimento da linha elétrica temos o valor de $N_{L}$ dobrado.

$$
N_{L}=N_{G} \cdot A_{L} \cdot C_{I} \cdot C_{E} \cdot C_{T} \cdot 10^{-6}
$$

Onde:

$N_{L}$ é o número de sobretensões de amplitude não inferior a $1 \mathrm{kV}$;

$N_{G}$ é a densidade de descargas atmosféricas para a terra $\left(1 / \mathrm{km}^{2} \mathrm{x}\right.$ ano);

$A_{L}$ é a área de exposição equivalente de descargas atmosféricas que atinge a linha, em $\mathrm{m}^{2}$.

$C_{I}$ é o fator de instalação da linha;

$C_{T}$ é o fator tipo de linha;

$C_{E}$ é o fator ambiental.

A última avaliação que deve ser feita é em relação ao número médio anual de eventos perigosos devido a descargas atmosféricas perto da linha, $N_{I}$, a expressão utilizada para esse parâmetro pode ser vista na equação (6). Após a substituição dos dados o resultado foi 10,4, admitindo que o comprimento da linha de telecomunicações será igual ao comprimento da linha elétrica temos o valor de $N_{I}$ dobrado.

$$
N_{I}=N_{G} \cdot A_{I} \cdot C_{I} \cdot C_{E} \cdot C_{T} \cdot 10^{-6}
$$

Onde:

$N_{I}$ é o número de sobretensões de amplitude não inferior a $1 \mathrm{kV}$;

$N_{G}$ é a densidade de descargas atmosféricas para a terra $\left(1 / \mathrm{km}^{2} \mathrm{x}\right.$ ano);

$A_{I}$ é a área de exposição equivalente de descargas atmosféricas perto da linha, em $\mathrm{m}^{2}$. 
$C_{I}$ é o fator de instalação da linha;

$C_{T}$ é o fator tipo de linha;

$C_{E}$ é o fator ambiental.

\subsubsection{Avaliação da probabilidade de danos}

A probabilidade de um choque elétrico causar ferimentos a seres vivos, $P_{A}$, é avaliado por meio da expressão (7), o valor de $P_{A}$ encontrado foi 1. $P_{B}$, se refere a probabilidade de uma descarga atmosférica em uma estrutura causar danos físicos.

$$
P_{A}=P_{T A} \cdot P_{B}
$$

Onde:

$P_{A}$ é a probabilidade de uma descarga atmosférica em uma estrutura causar ferimentos a seres vivos;

$P_{T A}$ esse fator depende das medidas de proteção contra tensões de toque e passo;

$P_{B}$ depende do nível de proteção contra descargas atmosféricas.

Os valores da probabilidade de uma descarga atmosférica em uma linha causar ferimentos a seres vivos por choque elétrico $\left(P_{U}\right)$, está diretamente ligado as características de blindagem da linha, avisos de restrições e utilização de DPS. Essa probabilidade é expressa pela função (8), que ao substituir os valores, o resultado encontrado foi 1.

$$
P_{U}=P_{T U} \cdot P_{E B} \cdot P_{L D} \cdot C_{L D}
$$

Onde:

$P_{U}$ é a probabilidade de uma descarga atmosférica na linha causar ferimentos;

$P_{T U}$ depende das medidas de proteção contra tensões de toque;

$P_{E B}$ depende das ligações equipotenciais para descargas atmosféricas;

$P_{L D}$ é a probabilidade de falhas no sistema devido a descargas atmosféricas;

$C_{L D}$ é o fator que depende da blindagem.

Os valores da probabilidade de uma descarga atmosférica em uma linha causar danos físicos $\left(P_{V}\right)$, é dado pela equação (9) e está diretamente ligada as características da blindagem da linha, da tensão de impulso dos sistemas conectados a ela ou do uso de DPS. Após a substituição dos valores na equação o resultado obtido foi 1.

$$
P_{V}=P_{E B} \cdot P_{L D} \cdot C_{L D}
$$

Onde: 
$P_{V}$ é a probabilidade de uma descarga atmosférica na linha causar danos físicos;

$P_{E B}$ depende das ligações equipotencias para descargas atmosféricas;

$P_{L D}$ é a probabilidade de falhas no sistema devido a descargas atmosféricas;

$C_{L D}$ é o fator que depende da blindagem.

\subsubsection{Avaliação da quantidade de perdas}

O valor da perda está diretamente relacionado ao tipo de perda associado. A perda se refere a quantidade relativa média de um tipo particular de dano para um evento perigoso. A L1, referente a perda de vida humana, é o foco deste trabalho. Com isso, os valores para cada tipo de zona são representados pelas equações (10) e (11), onde substituindo seus valores, o resultado obtido respectivamente foram $6,8493 \times 10^{-5}$ e $1,3699 \times 10^{-5}$.

$$
L_{A}=L_{U}=r_{t} \cdot L_{T} \cdot \frac{n_{Z}}{n_{t}} \cdot \frac{t_{Z}}{8760}
$$

Onde:

$L_{T}$ é o número médio típico de vítimas por choque elétrico;

$r_{t}$ é um fator de redução da perda de vida humana dependendo do tipo de solo;

$n_{z}$ é o número de pessoas na zona;

$n_{t}$ é o número total de pessoas na estrutura;

$t_{z}$ é o tempo que as pessoas ficam na zona, em horas por ano.

$$
L_{B}=L_{V}=r_{p} \cdot r_{f} \cdot h_{z} \cdot L_{F} \cdot \frac{n_{Z}}{n_{t}} \cdot \frac{t_{Z}}{8760}
$$

Onde:

$L_{F}$ é o número médio típico de vítimas por danos físicos;

$r_{p}$ é um fator de redução da perda de vida humana dependendo das providências tomadas contra incêndio;

$r_{f}$ é um fator de redução da perda devido a danos físicos dependendo do risco de incêndio;

$h_{z}$ é um fator de aumento da perda devido a danos físicos quando um perigo especial estiver presente;

$n_{z}$ é o número de pessoas na zona;

$n_{t}$ é o número total de pessoas na estrutura;

$t_{z}$ é o tempo que as pessoas ficam na zona, em horas por ano.

2.1.5. Avaliação geral do risco e seleção das medidas de proteção 
A avaliação geral de risco é calculada utilizando o número de eventos perigosos, probabilidade de danos e quantidade de perdas. O risco R1, é a soma de seus componentes e é dado pela expressão (12), que corresponde ao risco de perda de vida humana. As componentes $R_{A}, R_{B}, R_{U}$ e $R_{V}$ são expressos pelas funções (13), (14), (15) e (16), e a Tabela 4 mostra os resultados obtidos.

$$
\begin{gathered}
R_{1}=R_{A}+R_{B}+R_{U}+R_{V} \\
R_{A}=N_{D} \cdot P_{A} \cdot L_{A} \\
R_{B}=N_{D} \cdot P_{B} \cdot L_{B} \\
R_{U}=\left(N_{L}+N_{D J}\right) \cdot P_{U} \cdot L_{U} \\
R_{V}=\left(N_{L}+N_{D J}\right) \cdot P_{V} \cdot L_{V}
\end{gathered}
$$

Tabela 4 - Componentes de risco da estrutura atual

\begin{tabular}{c|c}
\hline Componentes do risco & Resultado \\
\hline$R_{A}$ & $1,9452 \times 10^{-6}$ \\
\hline$R_{B}$ & $3,8905 \times 10^{-7}$ \\
\hline$R_{U}$ & $7,1233 \times 10^{-6}$ \\
\hline$R_{V}$ & $1,4247 \times 10^{-6}$ \\
\hline \multicolumn{2}{c}{ Fonte: (Os autores, 2019) }
\end{tabular}

O valor de R1 encontrado foi de $1,0882 \times 10^{-5}$, que é superior ao permitido pela norma, sendo assim, se torna necessário a implementação de um sistema de proteção. Para reduzir o risco a valores aceitáveis é necessário atingir os fatores mais críticos descritos pela análise, sendo assim, destaca-se a adoção de um sistema de proteção contra descargas atmosféricas classe III, conseguindo neutralizar $R_{A}$ e $R_{B}$ através da redução da probabilidade $P_{B}$. Podemos observar os novos valores pertinentes aos riscos após a implementação de um sistema de proteção na Tabela 5. 
Tabela 5 - Componentes de risco após SPDA classe III

\begin{tabular}{c|c}
\hline Componentes do risco & Resultado \\
\hline$R_{A}$ & $1,9452 \times 10^{-7}$ \\
\hline$R_{B}$ & $3,8905 \times 10^{-8}$ \\
\hline$R_{U}$ & $7,1233 \times 10^{-6}$ \\
\hline$R_{V}$ & $1,4247 \times 10^{-6}$ \\
\hline $\mathrm{R} 1$ & $8,7814 \times 10^{-6}$ \\
\hline \multicolumn{2}{|c|}{ Fonte: (Os autores, 2019) }
\end{tabular}

\subsection{Projeto do SPDA}

O sistema de proteção contra descargas atmosféricas utilizado, será com base no método das malhas ou método de Faraday, considerando um nível de proteção classe III, assim trazendo a edificação para níveis aceitáveis de proteção. Vale ressaltar que esse projeto será desenvolvido de acordo com as normas vigentes.

\subsubsection{Posicionamento e dimensionamento do subsistema captor}

A área da edificação em que esse trabalho se baseia é de $1448,58 \mathrm{~m}^{2}$, essa é uma informação muito importante para se projetar uma boa malha de captação, de acordo com a (NBR 5419, 2015) o subsistema captor de um SPDA classe III, o espaçamento máximo admitido entre os condutores da malha não deve ultrapassar 15 metros, como indicado na Tabela 6.

Tabela 6 - Valores de parâmetros de diferentes métodos de proteção

\begin{tabular}{c|c|c}
\hline \multirow{2}{*}{ Classe do SPDA } & \multicolumn{2}{|c}{ Métodos de proteção } \\
\cline { 2 - 3 } & Máximo afastamento dos condutores da malha & Raio esfera rolante \\
\hline I & $5 \times 5$ & 20 \\
\hline II & $10 \times 10$ & 30 \\
\hline III & $15 \times 15$ & 45 \\
\hline IV & $20 \times 20$ & 60 \\
\hline
\end{tabular}

Fonte: Adaptado de (NBR 5419, 2015)

O número de condutores da malha captora pode ser calculado utilizando a equação (17), com isso pode-se dimensionar a quantidade de cabos que formam a malha e qual a melhor posição dos cabos encordoados de cobre, de uma maneira que atenda a norma. Encontra-se assim que no maior comprimento da construção, serão necessários 5 condutores e no menor comprimento da edificação, serão necessários 3 condutores, formando assim a malha de captação. Entretanto, estes são os valores mínimos e neste projeto, para a malha de captação preferiu-se utilizar 6 condutores no maior comprimento e 4 condutores no menor comprimento, além de um condutor circulando todo o perímetro do beiral (Figura 9). 


$$
N_{c m}=\frac{L_{m}}{D_{c m}}+1
$$

Onde:

$N_{c m}$ é o número de condutores na malha;

$L_{m}$ é o comprimento em metro;

$D_{c m}$ é a dimensão máxima da malha.

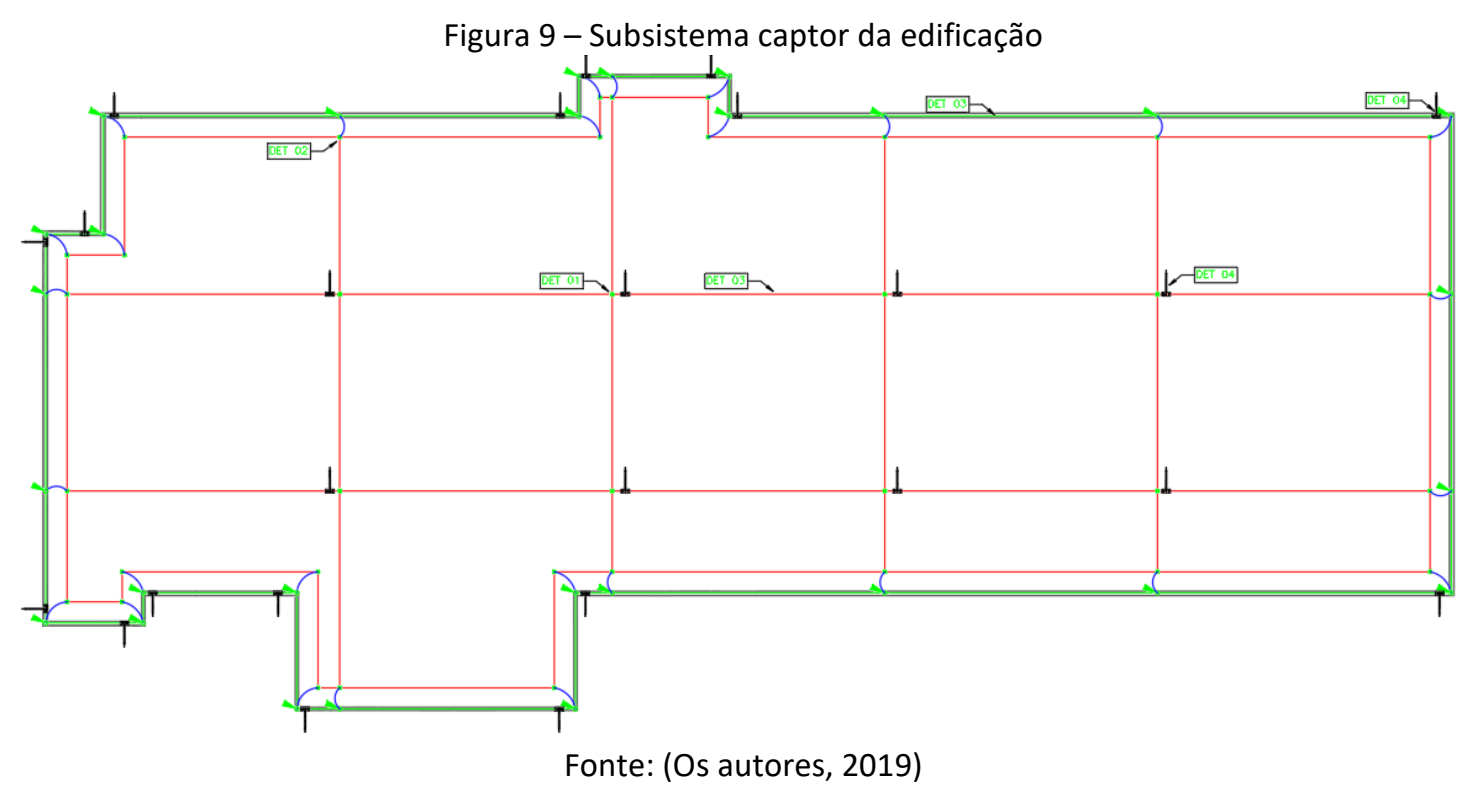

2.2.2. Posicionamento e dimensionamento do subsistema de descida

A (NBR 5419, 2015) especifica que a distância máxima entre os condutores de descida seja de 15 metros, como se pode observar na Tabela 7, isso para SPDA classe III, utilizando o método de Faraday. A equação (18) é usada para que se possa dimensionar de maneira mais precisa a quantidade de descidas a fim de atender a norma supra citada. Com a utilização dessa equação, chegou-se ao valor de 12 descidas assim atendendo-se os requisitos mínimos da norma. Adotou-se o mesmo material utilizado no subsistema captor, ou seja, cabo de cobre encordoado.

Tabela 7 - Espaçamentos típicos entre os condutores
\begin{tabular}{c|c}
\hline Classe do SPDA & Espaçamento em m \\
\hline I & 10 \\
\hline II & 15 \\
\hline III & 15 \\
\hline IV & 20 \\
\hline \\
$N_{c d}=\frac{P_{c o}}{D_{c d}}$
\end{tabular}

Onde: 
$N_{c d}$ é o número de descidas;

$P_{c o}$ é o perímetro em metros;

$D_{c d}$ é a distância máxima entre as descidas.

Neste projeto optou-se por usar uma maior quantidade de descidas visto que desta forma haveria um padrão mais linear entre as distâncias, como se pode ser visto na Figura 10. A utilização das descidas conforme dito, será de cobre nu encordoado, haja visto que quando se chegar a uma distância de três metros do solo o mesmo entrará em um eletroduto de PVC de 1" com uma caixa de passagem para a inspeção da conexão do subsistema de descida com o subsistema de aterramento.

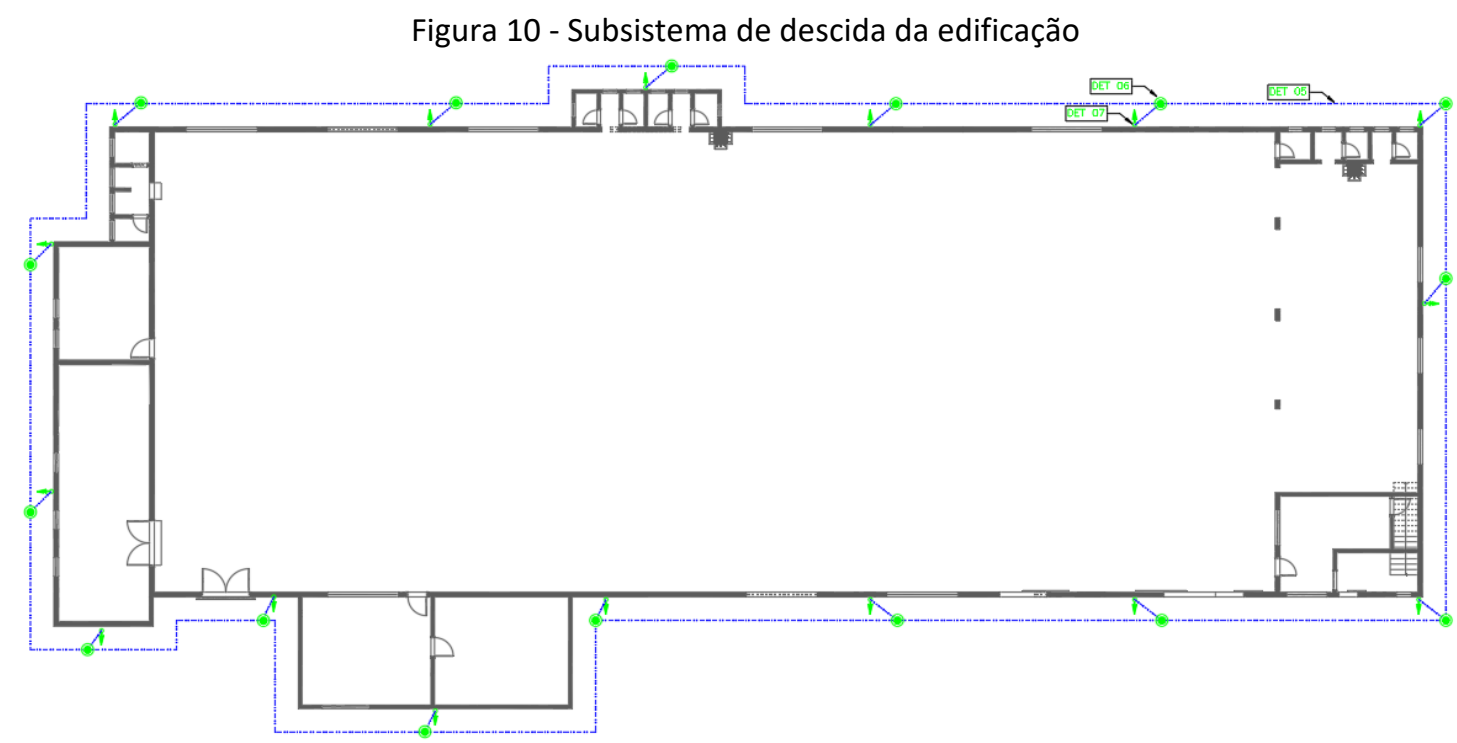

Fonte: (Os autores, 2019)

\subsubsection{Aterramento}

Para a malha de aterramento, por se tratar de um sistema de proteção contra descargas atmosféricas classe III, não se exige grandes considerações. Desta forma, foi adotado de acordo com a norma hastes de aterramento de 2,4 metros, sendo uma para cada descida, com isso serão necessárias 16 hastes de aterramento em malha conectadas por um cabo de cobre nu encordoado com bitola de $50 \mathrm{~mm}^{2}$, enterrados a uma profundidade de $50 \mathrm{~cm}$, e espaçado da edificação em 1 metro.

\subsubsection{Custo do projeto}

Os materiais utilizados, a quantidade, o preço unitário e o preço total de cada item dos subsistemas do projeto, podem ser vistos da Tabela 8 à Tabela 10 . 0 custo total do projeto pode ser visto na Tabela 11. 
Tabela 8 - Descrição e valor do material utilizado no subsistema captor

\begin{tabular}{|c|c|c|c|c|}
\hline Item & Descrição & Quant. & Preço unit. & Preço total \\
\hline 1 & Cabo de cobre Nú $35 \mathrm{~mm}^{2}$ & 522 & $\mathrm{R} \$ 21,00$ & $\mathrm{R} \$ 10.962,00$ \\
\hline 2 & Presilha de latão $35 \mathrm{~mm}^{2}$ & 500 & $\mathrm{R} \$ 1,38$ & $\mathrm{R} \$ 690,00$ \\
\hline 3 & Bucha nylon S8 & 548 & $\mathrm{R} \$ 0,17$ & $\mathrm{R} \$ 93,16$ \\
\hline 4 & Parafuso S8 & 548 & $R \$ 0,60$ & $\mathrm{R} \$ 328,80$ \\
\hline 5 & Conector de cruzamento $35 \mathrm{~mm}^{2}$ & 20 & $\mathrm{R} \$ 27,80$ & $\mathrm{R} \$ 556,00$ \\
\hline 6 & Conector de derivação $35 \mathrm{~mm}^{2}$ & 69 & $\mathrm{R} \$ 19,30$ & $\mathrm{R} \$ 1.331,70$ \\
\hline 7 & Minicaptor $35 \mathrm{~cm}$ aço galv. a quente & 24 & $R \$ 23,50$ & $\mathrm{R} \$ 564,00$ \\
\hline Total & & & & $R \$ 14.525,66$ \\
\hline
\end{tabular}

Fonte: (Os autores, 2019)

Tabela 9 - Descrição e valor do material utilizado no subsistema de descida

\begin{tabular}{|c|c|c|c|c|}
\hline Item & Descrição & Quant. & Preço unit. & Preço total \\
\hline 1 & Cabo de cobre Nú $35 \mathrm{~mm}^{2}$ & 104 & $\mathrm{R} \$ 21,00$ & $\mathrm{R} \$ 2.184,00$ \\
\hline 2 & Presilha de latão $35 \mathrm{~mm}^{2}$ & 104 & $\mathrm{R} \$ 1,38$ & $\mathrm{R} \$ 143,52$ \\
\hline 3 & Bucha nylon S8 & 104 & $\mathrm{R} \$ 0,17$ & $\mathrm{R} \$ 17,68$ \\
\hline 4 & Parafuso S8 & 104 & $\mathrm{R} \$ \mathbf{0 , 6 0}$ & $\mathrm{R} \$ 62,40$ \\
\hline 5 & Eletroduto de PVC 1" & 16 & $\mathrm{R} \$ 21,99$ & $\mathrm{R} \$ 351,84$ \\
\hline 6 & Conector de derivação $35 \mathrm{~mm}^{2}$ & 16 & $\mathrm{R} \$ 19,30$ & $\mathrm{R} \$ 308,80$ \\
\hline 7 & Abraçadeira de PVC 1" & 32 & $\mathrm{R} \$ 7,99$ & $\mathrm{R} \$ 255,68$ \\
\hline 8 & Conduíte de alumínio $1 "$ & 16 & $\mathrm{R} \$ 9,76$ & $\mathrm{R} \$ 156,16$ \\
\hline 9 & Conector de bronze & 16 & $\mathrm{R} \$ 14,98$ & $\mathrm{R} \$ 239,68$ \\
\hline 10 & Cabo de cobre NÚ $50 \mathrm{~mm}^{2}$ & 24 & $\mathrm{R} \$ 27,59$ & $\mathrm{R} \$ 662,16$ \\
\hline Total & & & & $\mathrm{R} \$ \mathbf{4 . 3 8 1 , 9 2}$ \\
\hline
\end{tabular}

Fonte: (Os autores, 2019)

Tabela 10 - Descrição e valor do material utilizado no aterramento

\begin{tabular}{|c|c|c|c|c|}
\hline Item & Descrição & Quant. & Preço unit. & Preço total \\
\hline 1 & Cabo de cobre nu $50 \mathrm{~mm}^{2}$ & 24 & $\mathrm{R} \$ 27,59$ & $R \$ 662,16$ \\
\hline 2 & Conector de bronze & 16 & $\mathrm{R} \$ 14,98$ & $\mathrm{R} \$ 239,68$ \\
\hline 3 & Caixa de aterramento & 16 & $\mathrm{R} \$ 14,99$ & $R \$ 239,84$ \\
\hline 4 & Haste de aterramento & 16 & $\mathrm{R} \$ \mathbf{6 9 , 9 0}$ & $\mathrm{R} \$ 1.118,40$ \\
\hline Total & & & & $\mathrm{R} \$ 2.260,08$ \\
\hline
\end{tabular}

Fonte: (Os autores, 2019)

Tabela 11 - Valor total do projeto

\begin{tabular}{c|c|c}
\hline Item & Descrição & Preço total \\
\hline $\mathbf{1}$ & Total subsistema captor & $\mathrm{R} \$ 14.525,66$ \\
\hline $\mathbf{2}$ & Total subsistema de descida & $\mathrm{R} \$ 4.381,92$ \\
\hline $\mathbf{3}$ & Total aterramento & $\mathrm{R} \$ 2.260,08$ \\
\hline Total Projeto & & $\mathbf{R} \mathbf{2 1 . 1 6 7 , 6 6}$ \\
\hline \multicolumn{3}{|c}{ Fonte: (Os autores, 2019) }
\end{tabular}




\section{CONSIDERAÇÕES FINAIS}

Por fim, conclui-se que é necessário a instalação do sistema de proteção aqui proposto, para que a edificação esteja em níveis de segurança e confiabilidade aceitáveis, e acima de tudo garanta a segurança das pessoas na edificação, uma vez que a análise de riscos mostrou que o galpão apresenta valores elevados de risco, principalmente em relação ao componentes RA, relacionado a ferimentos a seres vivos provocados por choque elétrico, e ao componente RU, relacionado a ferimentos aos seres vivos provocado por choque elétrico em linhas de transmissão, tais riscos vão para valores aceitáveis com a execução desse SPDA projetado no presente trabalho. Além disso, o trabalho indica que o custo, $\mathrm{R} \$ 21.167,66$ para a instalação desse sistema na edificação é inferior ao valor que uma possível falha poderia custar, tendo em vista o valor das máquinas, dos materiais e da paralização da produção.

\section{REFERENCIAS}

ARAÚJO, M. A. D. Projeto de um Sistema de Proteção Contra Descargas Atmosfericas para uma Linha de Transmissão de 69kV, São Carlos, 2010.

CREDER, H. Instalações Elétricas. 16. ed. Rio de Janeiro: LTC, 2016.

ELAT. ELAT - Grupo de Eletricidade Atmosférica. INPE, 2019. Disponivel em: <http://www.inpe.br/webelat/homepage/menu/protecao/protecao.edificacoes.php>. Acesso em: 05 jun. 2019.

FILHO, S. V. Descargas Atmosféricas - uma abordagem de engenharia. [S.I.]: Artliber, 2005.

FRAZÃO, D. Michael Faraday - Físico e químico inglês. eBiografia, 2019. Disponivel em: <https://www.ebiografia.com/michael_faraday/>. Acesso em: 07 jun. 2019.

KINDERMANN, G. Aterramento Elétrico. Porto Alegre: Sagra, 1995.

MATTOS, E. V. Relações das Propriedades Físicas das Nuvens Convectivas com as Descargas Elétricas, São José dos Campos, 2009.

MINISTÉRIO DA CIDADANIA. Blog com Ciência. MuseuWEG de Ciência e Tecnologia, 2019. Disponivel em: <https://museuweg.net/blog/benjamin-franklin-ciencia-e-eletricidade/>. Acesso em: 07 jun. 2019.

NBR 5419. NBR 5419 - Proteção de estruturas contra descargas atmosféricas. [S.I.]: ABNT, 2015.

NOLETO, S. R. C. As Estruturas Metálicas das Edificações como Sistema de Proteção contra Descargas Atmosféricas. Monografia de Graduação, Brasília, p. 124, dez. 2006. 
STÉFANI, R. V. D. Metodologia de Projeto de Sistemas de Proteção Contra Descargas Atmosféricas para Edifício Residencial. São Carlos: [s.n.], 2011.

SUETA, H. E. O gerenciamento de risco segundo a Parte 2 da ABNT NBR 5419. Espaço 5419, v. 109, fev. 2015. Disponivel em: <https://www.osetoreletrico.com.br/o-gerenciamento-de-risco-segundoa-parte-2-da-abnt-nbr-5419/>. Acesso em: 06 jun. 2019.

VAZQUEZ, R. Blog de Engenharia. Fluxo Consultoria, 13 ago. 2018. Disponivel em: <https://fluxoconsultoria.poli.ufrj.br/blog/\%20arquitetura-construcao/spda-do-condominio/>. Acesso em: 04 jun. 2019.

VEIGA, J. E. D. Aquecimento Global - frias contendas cientifícas. [S.I.]: SENAC, 2008. 